\title{
Does dietary folic acid supplementation in mouse NTD models affect neural tube development or gamete preference at fertilization?
}

\author{
Ghunwa A Nakouzi ${ }^{1,2}$ and Joseph H Nadeau ${ }^{1,3^{*}}$
}

\begin{abstract}
Background: Neural tube defects (NTDs) are the second most common birth defect in humans. Dietary folic acid (FA) supplementation effectively and safely reduces the incidence of these often debilitating congenital anomalies. FA plays an established role in folate and homocysteine metabolism, but the means by which it suppresses occurrence of NTDs is not understood. In addition, many cases remain resistant to the beneficial effects of folic acid supplementation. To better understand the molecular, biochemical and developmental mechanisms by which FA exerts its effect on NTDs, characterized mouse models are needed that have a defined genetic basis and known response to dietary supplementation.

Results: We examined the effect of FA supplementation, at 5-fold the level in the control diet, on the NTD and vertebral phenotypes in $A p o b^{t m I U n c}$ and Vangl2 $2^{L p}$ mice, hereafter referred to as Apob and $L p$ respectively. The FA supplemented diet did not reduce the incidence or severity of NTDs in Apob or Lp mutant homozygotes or the loop-tail phenotype in $L p$ mutant heterozygotes, suggesting that mice with these mutant alleles are resistant to FA supplementation. Folic acid supplementation also did not affect the rate of resorptions or the size of litters, but instead skewed the embryonic genotype distribution in favor of wild-type alleles.

Conclusion: Similar genotypic biases have been reported for several NTD models, but were interpreted as dietinduced increases in the incidence and severity of NTDs that led to increased embryonic lethality. Absence of differences in resorption rates and litter sizes argue against induced embryonic lethality. We suggest an alternative interpretation, namely that FA supplementation led to strongly skewed allelic inheritance, perhaps from disturbances in polyamine metabolism that biases fertilization in favor of wild-type gametes.
\end{abstract}

Keywords: Neural tube defects, Mouse models, Folic acid, Apob, Vangl2, Embryonic lethality, Polyamines, Fertilization, Gametes, Sperm, Oocyte, Epigenetics

\section{Background}

Neural tube closure is an early developmental process that gives rise to the central nervous system, including the spinal cord and brain [1,2]. Failure of the neural tube to close properly leads to different clinical types of NTDs depending on the site and timing of closure failure [1-6]. Neural tube defects (NTDs) are serious and common birth defects resulting from both genetic and

\footnotetext{
* Correspondence: jnadeau@pnri.org

'Department of Genetics, Case Western Reserve University School of Medicine, Cleveland, OH, USA

${ }^{3}$ Present address: Pacific Northwest Research Institute, 720 Broadway, Seattle, WA 98122, USA

Full list of author information is available at the end of the article
}

environmental factors [1-6]. In humans, FA supplementation of maternal diet before and during pregnancy significantly reduces NTD incidence [1-8].

FA plays a role in both the folate cycle for the production of thymidylate and purines mediating cell division, and in the methylation cycle of homocysteine metabolism resulting in epigenetic regulation of gene expression [9-11]. Although the efficacy of FA supplementation is widely accepted, the mechanism by which FA reduces the incidence of NTDs is not understood and whether FA-resistant cases respond to alternative dietary nutrients is not generally known. Several studies have implicated FA in reproduction and fertility in humans [12-17] as well as with developmental delay and increased rates 
of cardiac defects and other fetal anomalies in mouse models [18-20], suggesting that the effects of folate metabolism and FA supplementation on pregnancy and gamete biology may be more diverse than generally appreciated.

Mouse NTD models with specific responses to different nutritional supplements can be used to study mechanisms of FA responsiveness in humans and mice, and to identify alternative approaches to prevent FA-resistant NTDs [21-23]. In particular, mouse models involving known genes, characterized mechanisms, and established responses to FA supplementation are needed. However, among more than 240 NTD mouse mutants and strains, only 19 have been tested for response to FA supplementation on the outcome of NTDs, with supplementation effective in some mutants but not others [2,23-26]. Our lab sought to expand this body of knowledge by studying the effect of FA on selected NTD mouse models.

We examined the FA response of two NTD mouse mutants. Apolipoprotein B (apoB) is a key structural component of several lipoproteins that transport circulating cholesterol, lipids, and vitamin E [27]. The $A p o b^{\text {tm1Unc }}$ mutant is the result of a genetically engineered loss-offunction (LOF) mutation in the $A p o b$ gene [27]. Apob homozygous embryos show a $30 \%$ penetrance of exencephaly alone or accompanied with hydrocephalus [27], see also [28] (Figure 1A vs B). By 8 weeks of age, mutants that have a closed neural tube show hydrocephalus in $32 \%$ of homozygotes and in $1 \%$ of heterozygotes. VANGL2 protein is one of two highly conserved membrane proteins involved in establishing planar cell polarity (PCP) and in regulating convergent extension movements during embryogenesis [29]. The Vangl2 $2^{L p}$ mutant results from a spontaneous LOF mutation in the Vangl2 gene [29], see also [28]. Lp homozygous embryos have a $100 \%$ penetrance of craniorachischisis due to failure to initiate neural tube closure at embryonic day E8.5 [30] (Figure 1A vs C). This mutation is inherited in a co-dominant manner and the heterozygous phenotype is characterized by a looped tail resulting from vertebral anomalies [29] (Figure 1A vs D). Neither mutant has been previously tested for response to dietary FA supplementation.

During our work on the effects of dietary FA supplementation on mouse models of NTDs, we made an observation that others had made with other NTD models, but were led to an alternative interpretation that seems more consistent with the entire body of data. In particular, we found that parental FA supplementation did not reduce the incidence or severity of NTDs in these two mouse models, but instead caused a substantial deficiency in the numbers of homozygous and heterozygous mutant embryos, without a corresponding increase in resorptions or a reduction in litter size. We suggest that FA supplementation led to preferential fertilization and biased segregation in heterozygous mutant mice. Obviously more work is needed to characterize molecular mechanisms, but we thought an initial report was appropriate to highlight this issue.

\section{Results}

We began by testing whether parental FA supplementation reduced the incidence or severity of NTDs in homozygous mutant embryos or the loop-tail phenotype in $L p$ heterozygous mutant mice. Timed-pregnancies were generated with females that were either supplemented with FA (10 ppm) or maintained on a baseline FA diet (2 ppm) before mating and during pregnancy. Homozygous $A p o b$ and $L p$ embryos were examined for NTDs [27-29], see also [31-33] and $L p$ mutant heterozygotes for the loop-tail phenotype (Figure 1). In particular, the proportion of affected embryos did not differ between the two test and control groups (Table 1), suggesting that these NTD mutants are resistant to the beneficial effects of dietary FA supplementation.

Unexpectedly, both supplemented lines showed a substantial deficiency of homozygous and heterozygous mutant embryos at the higher FA concentration. Because these single gene mutations are inherited in a Mendelian manner [27-29], 25\% of the embryos are expected to have a wild-type genotype $(+/+), 50 \%$ a heterozygous
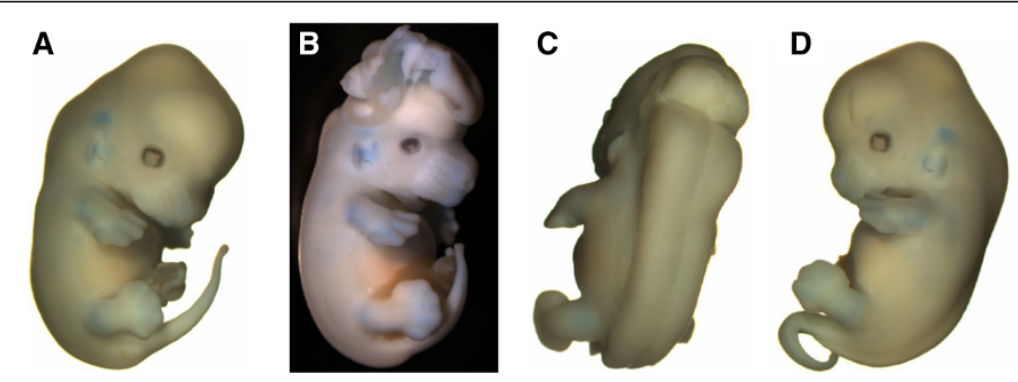

Figure 1 Examples of congenital defects in Apob and $L p$ mutant mice. A. Normal embryo, B. Apob - exencephaly, C. $L p-c r a n i o r a c h i s c h o s i s$, and D. Lp - loop-tail. 
Table 1 Association between parental FA supplementation and incidence of NTDs

\begin{tabular}{|c|c|c|}
\hline Mutant, diet & $\%$ Affected (n) & Sample size \\
\hline \multicolumn{3}{|c|}{$\overline{A p o b^{t m} 1 U n c / t m i U n c}$ - exencephaly } \\
\hline 2 ppm & $96(26)$ & 27 \\
\hline 10 ppm & $96(26)$ & 27 \\
\hline \multicolumn{3}{|c|}{ Vang/2 $2^{L / L P}$ - craniorachischisis } \\
\hline $2 \mathrm{ppm}$ & $100(15)$ & 15 \\
\hline 10 ppm & $100(11)$ & 11 \\
\hline \multicolumn{3}{|c|}{$\overline{V a n g} / 2^{L p / L p}-$ looped tail } \\
\hline $2 \mathrm{ppm}$ & $98(40)$ & 41 \\
\hline 10 ppm & $97(31)$ & 32 \\
\hline
\end{tabular}

Fisher's exact test was used to determine whether FA-supplementation affected the incidence of NTDs in mutant homozygotes or heterozygotes. The number of affected embryos is shown in parentheses. No significant differences were detected.

genotype (+/mutant), and $25 \%$ a homozygous mutant genotype (mutant/mutant) (Figure 2). The genotype distribution on the control diet was consistent with Mendelian expectations for both mutants, showing that segregation was normal at 2 ppm FA. By contrast, a clear deficiency was found for homozygous and heterozygous embryos conceived and maintained on 10 ppm FA (Table 2). For the supplemented $L p$ mutant, although the deviation from Mendelian ratios was not statistically significant, the observed numbers of homozygous and heterozygous mutant embryos was strongly reduced relative to expectations, with the percent difference comparable to results for the Apob mutant, but with a slightly smaller sample size (Table 2).

Finally, we sought to estimate the number of missing embryos. Because FA supplementation is not expected to affect the number of wild-type embryos, we accepted the number of $+/+$ embryos as indicative of Mendelian expectations. By extrapolation, we then estimated the expected number of heterozygous and homozygous mutants cf. $[24,25]$. This analysis assumed that fertilization was random with respect to the genetic constitution of gametes in both parents. We found that on the $10 \mathrm{ppm}$ diet, 52.5\% and $32.5 \%$ of the expected numbers of Apob mutant heterozygotes and homozygotes were missing, respectively (Table 2). Similarly, $30.4 \%$ and $52.2 \%$ of the expected numbers Lp mutant heterozygote and homozygote embryos were missing on the $10 \mathrm{ppm}$ diet. Interestingly, we found no evidence for increased rates of congenital anomalies among heterozygous mutant embryos on the supplemented diet, implying that a substantial number of phenotypically normal heterozygous mutant embryos were missing (Table 2). Finally, we noted that average litter size did not differ between test and control crosses. We also counted the number of resorptions as a measure of fetal loss, but these counts did not differ. Thus a substantial number of

\section{A. Observed genotype ratio $+/+:+/ \mathrm{m}: \mathrm{m} / \mathrm{m}$$$
p^{2}: 2 p q: q^{2}
$$ \\ Fertilization bias Punnett square $\pm \underline{m}$ p q None \begin{tabular}{ll|l|}
+ l & $p p$ & $q p$ \\
\hline
\end{tabular} $\mathrm{m} / \mathrm{q} \quad \mathrm{pq} p \mathrm{pp}$ \\ B. Observed
genotype ratio
$+/+:+/ \mathrm{m}: \mathrm{m} / \mathrm{m}$ \\ Fertilization bias None
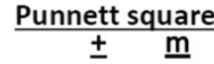 \\ $0.25: 0.50: 0.25$ \\ Case 1 \\ \begin{tabular}{c}
+0.50 .250 .25 \\
\hline
\end{tabular} \\ \begin{tabular}{ll|l|}
\hline m 0.5 & 0.25 & 0.25 \\
\hline
\end{tabular} \\ Case $20.45: 0.50: 0.05$ \\ Bias in one gender

\begin{tabular}{c|c|c|}
\multicolumn{1}{c}{} & \multicolumn{1}{c}{0.9} & 0.1 \\
\hline$+\mid 0.5$ & 0.45 & 0.05 \\
\cline { 3 - 3 } $\mathrm{m} \mid 0.5$ & 0.45 & 0.05
\end{tabular} \\ $\underline{\text { Case } 3} 0.49: 0.42: 0.09$ Bias in both genders

\begin{tabular}{c|c|c|}
\multicolumn{1}{c}{} & \multicolumn{1}{c}{0.7} & 0.3 \\
\cline { 3 - 4 }+0.7 & 0.49 & 0.21 \\
\cline { 2 - 3 } $\mathrm{m} 0.3$ & 0.21 & 0.09 \\
& &
\end{tabular}

Figure 2 Gamete bias at fertilization and conceptus genotype frequencies. ' $t$ ' and ' $m$ ' designate gametes that carry the wild-type or the mutant allele, respectively. Gamete frequencies are shown on the sides of the matrix, and conceptus genotype in the cells of the matrix. Each side of the matrix represents one of sexes in each mating. A. General case, where $p$ and $q$ denote alternative alleles. B. Arbitrary numbers were used to illustrate the consequences of gametic bias. Note that all eggs are fertilized and litter size remains unchanged in each scenario; only the genotypic ratio changes. 
Table 2 Embryo loss among progeny of NTD heterozygous mutant intercrosses

\begin{tabular}{|c|c|c|c|c|c|c|c|c|}
\hline \multirow[t]{2}{*}{ Mutant, Diet } & \multicolumn{3}{|c|}{ Obs. no. embryos } & \multirow[t]{2}{*}{ P-value $\left(x^{2}\right)$} & \multirow{2}{*}{$\begin{array}{l}\text { \% Lost heterozygous, } \\
\text { homozygous }\end{array}$} & \multirow{2}{*}{$\begin{array}{l}\text { \% Lost } \\
\text { (combined) }\end{array}$} & \multirow{2}{*}{$\begin{array}{l}\% \text { resorbed } \\
\text { (n) }\end{array}$} & \multirow{2}{*}{$\begin{array}{l}\text { Ave. litter size } \\
\text { (n) }\end{array}$} \\
\hline & $+/+$ & $+/-$ & $-1-$ & & & & & \\
\hline \multicolumn{9}{|l|}{$A p o b^{\text {tm1Unc }}$} \\
\hline 2 ppm & 24 & 48 & 27 & ns & - & - & $12.3(14)$ & $6.2(16)$ \\
\hline 10 ppm & 40 & 38 & 27 & $0.004(11.2)$ & $52.5,32.5$ & 45.8 & $7.8(9)$ & $5.8(18)$ \\
\hline \multicolumn{9}{|l|}{ Vangl2 $2^{L p}$} \\
\hline $2 \mathrm{ppm}$ & 13 & 41 & 15 & ns & - & - & $9.2(7)$ & $4.3(16)$ \\
\hline 10 ppm & 23 & 32 & 11 & $0.11(4.4)$ & $(30.4,52.2)$ & 37.7 & $9.6(7)$ & $4.4(15)$ \\
\hline
\end{tabular}

All embryos were genotyped. Only genotyped embryos were included in litter size metrics. Resorptions were not genotyped. Chi-square goodness-of-fit tests were used to determine whether the observed genotypic distribution of embryos deviated significantly from Mendelian expectations (1:2:1) for the two NTD models and for the two FA diets. The P-value for this test is provided. To calculate the percentage of "embryo loss", we assumed that the observed number of wild-type embryos was the correct number for the 1:2:1 Mendelian distribution. From this, we estimated expected numbers of heterozygous and mutant homozygous embryos, and then calculated the difference between the expected and observed numbers. The percent embryo loss was calculated for Apob (10 ppm) where genotyping results differed significantly from Mendelian expectations (bold numbers). The percent embryo loss is also provided in parentheses for Vangl2 where a strong but non-significant trend was found. Note that differences in resorptions and litter size did not account for percent embryo loss. Bold numbers highlight results of particular interest. ns, not significant; na, not applicable.

embryos appeared to be missing, with no evidence for embryonic lethality.

\section{Discussion}

Understanding the molecular and developmental mechanisms by which dietary supplementation affects neural tube development is critical to reducing the impact of one of the most common birth defects, especially since some NTDs appear to be resistant to the beneficial effects of FA [1-6,21-23]. Animal models are essential for studying experimentally the ways that these dietary factors modulate protein functions, biochemical pathways, and developmental processes during neural tube formation [21-26,28,34]. In the present study, we found that parental FA supplementation did not protect embryos either from exencephaly in $\mathrm{Apob}^{-/-}$embryos or from craniorachischisis and looped-tail phenotypes in $L p^{-/-}$and $L p^{+/-}$embryos (Table 1). Embryos exposed to the test diet that had 5-fold more FA than the control diet did not show significant changes in the incidence or severity of defects. Thus FA supplementation did not beneficially impact aspects of lipid transport (Apob) and planar cell polarity (Vangl2) in these two mutant mice.

Unexpectedly, we found strongly biased genotype distributions with folate supplementation in both mutants, but without reduced litter size, increased resorption rates, or other evidence for differences in embryonic viability. We reviewed the literature to determine whether similar genotypic deviations without embryo loss had been reported in other NTD diet-supplementation studies. Responses of several NTD models to various nutrients have been tested, with some showing responsiveness and others resistance to supplementation or to deprivation [21-26,28,34]. Data in some reports are consistent with normal Mendelian segregation in both test and control groups [18-20,23,25,34]. Remarkably, at least two studies involving three NTDs models also report non-Mendelian segregation (Table 3). The proportion of missing embryos was similar among models and studies, with the observed genotypic deviations corresponding to a $\sim 20 \%-\sim 70 \%$ reduction in the number of both heterozygous and homozygous mutant embryos

Table 3 Embryo loss among progeny of NTD mutant intercrosses

\begin{tabular}{|c|c|c|c|c|c|c|c|c|c|}
\hline \multirow[t]{2}{*}{ Mutant, Diet } & \multicolumn{3}{|c|}{ Obs. no. embryos } & \multirow[t]{2}{*}{ P-value $\left(x^{2}\right)$} & \multirow{2}{*}{$\begin{array}{l}\text { \% Lost heterozygous, } \\
\text { homozygous }\end{array}$} & \multirow{2}{*}{$\begin{array}{l}\text { \% Lost } \\
\text { (combined) }\end{array}$} & \multirow{2}{*}{$\begin{array}{l}\text { \% Resorbed } \\
\text { (n) }\end{array}$} & \multirow{2}{*}{$\begin{array}{l}\text { Ave. litter size }{ }^{* *} \\
\text { (n) }\end{array}$} & \multirow[t]{2}{*}{ Reference* } \\
\hline & $+/+$ & $+/-$ & $-1-$ & & & & & & \\
\hline \multicolumn{10}{|l|}{$\operatorname{Lrp6}^{k o}$} \\
\hline $2 \mathrm{ppm}$ & 37 & 73 & 20 & $0.04(6.4)$ & $1.4,45.9$ & na & $18(29)$ & $7.9(20 ; 4,12)$ & 1 \\
\hline 10 ppm & 55 & 86 & 16 & $0.0001(20.8)$ & $21.8,70.1$ & 38.2 & $16(30)$ & $8.5(22 ; 2-12)$ & 1 \\
\hline \multicolumn{10}{|l|}{ Zic2 } \\
\hline $2 \mathrm{ppm}$ & 56 & 84 & 32 & $0.03(6.8)$ & $25.0,42.9$ & 31.0 & $7.5(14)$ & $6.1(28)$ & 2 \\
\hline 10 ppm & 38 & 98 & 36 & ns & na & na & $11.8(23)$ & $5.7(30)$ & 2 \\
\hline \multicolumn{10}{|l|}{$L 3 P$} \\
\hline $2 \mathrm{ppm}$ & 28 & 40 & 11 & $0.03(7.3)$ & $28.6,60.7$ & 39.3 & $15.9(15)$ & $7.2(13)$ & 2 \\
\hline 10 ppm & 14 & 28 & 16 & ns & na & na & $18.3(13)$ & $4.2(10)$ & 2 \\
\hline
\end{tabular}

See Table 2 for details. ns - not significant, na - not applicable. (Revised from Gray et al. [24] and Marean et al. [25], with permission of the publishers) Bold numbers highlight results of particular interest.

*References: (1) Gray et al. [24], (2) Marean et al. [25]. ** Based on genotyped embryos only. 
(Table 3). In particular, embryo loss as a function of Mendelian expectations for homozygous mutants ranged from 43\% for Zic2 to 70\% for Lrp6, and for heterozygous mutants from $22 \%$ for $L r p 6$ to $29 \%$ for $L 3 P$. Interestingly, corresponding changes in resorptions rates and litter sizes were not found.

Significant departures from Mendelian expectations without embryo loss may be a regular but overlooked finding in NTD diet supplementation studies. Previously 19 models were tested for response to supplementation. The present study brings the total to 21. Of these five (Lrp6, Zic3, L3P, Apob and Vangl2) show non-Mendelian segregation (Tables 2 and 3), suggesting that skewed genotype ratios without embryonic lethality may be common. Treatment protocols differ among studies with supplementation in some cases introduced before conception e.g. [18-20,23-25] and in other cases during gestation e.g. $[23,33,34]$. Only the former protocol tests for effects of supplementation on Mendelian segregation.

Deficiency of particular genotypic classes is usually interpreted as diet-induced lethality among genetically predisposed embryos [24,25]. Various evidence argues against this interpretation. For example, fertilization of "reserve" wild-type oocytes might compensate for missing conceptuses. But with few exceptions, all ovulated oocytes are fertilized and litter size is closely related to the number of ovulated eggs. Results for heterozygotes are also particularly interesting because these mice usually show full viability, with $\operatorname{Vangl} 2^{L p /+}$ only showing a looped tail and $A p o b^{\text {tm } 1 U n c /+}$ heterozygotes appearing phenotypically normal $[27,29]$. We found no evidence for FA-induced congenital anomalies among surviving heterozygous embryos. Diet-induced anomalies are occasionally reported, e.g. an NTD in a single $\mathrm{Pax3}^{\mathrm{Sp} 2 \mathrm{H} /+}$ heterozygote that had been exposed to thymidine supplementation during gestation [23]. But these cases are exceptional and loss of substantial numbers of phenotypically normal mutant heterozygotes with dietary supplementation is therefore perplexing.

The epidemiological evidence for folate effects is largely based on differences in NTD occurrence in supplemented versus unsupplemented pregnancies [1-8]. Genetic tests are rarely included in these population studies because the genetic basis is not known for most NTD cases [1,6]. Hence the inference is made that a change in NTD occurrence results from beneficial effects of folate action on development of the neural tube, rather than a change in the occurrence of NTD-susceptible genotypes in FA supplemented populations.

We propose an alternative interpretation, namely that FA supplementation biases the combination of gametes that join to form a conceptus. Preferential fertilization would change the genotype distribution among conceptuses without reducing litter size or inducing embryonic lethality (Figure 2). We note that biased segregation was found only in intercrosses, and not in backcrosses to wild-type (GAN and JHN, unpubl.), suggesting a preference for specific combinations of sperm and oocyte at fertilization, rather than intrinsic gametic defects.

FA affects many aspects of reproduction and fertility as well as imprinting and related parent-of-origin effects. Anomalies in FA metabolism can affect fertility, placental function and pregnancy in humans [12-17] and in mice [35]. FA acid metabolism is actively involved in DNA methylation, a major class of epigenetic modification (see Figure 3 for a schematic of the relevant pathways). The one-carbon (folate) pathway involves acquisition of a methyl group from diet or metabolic salvage, and then its transfer to S-adenosylmethionine (SAM) in the methylation (homocysteine) pathway. SAM is the one-carbon

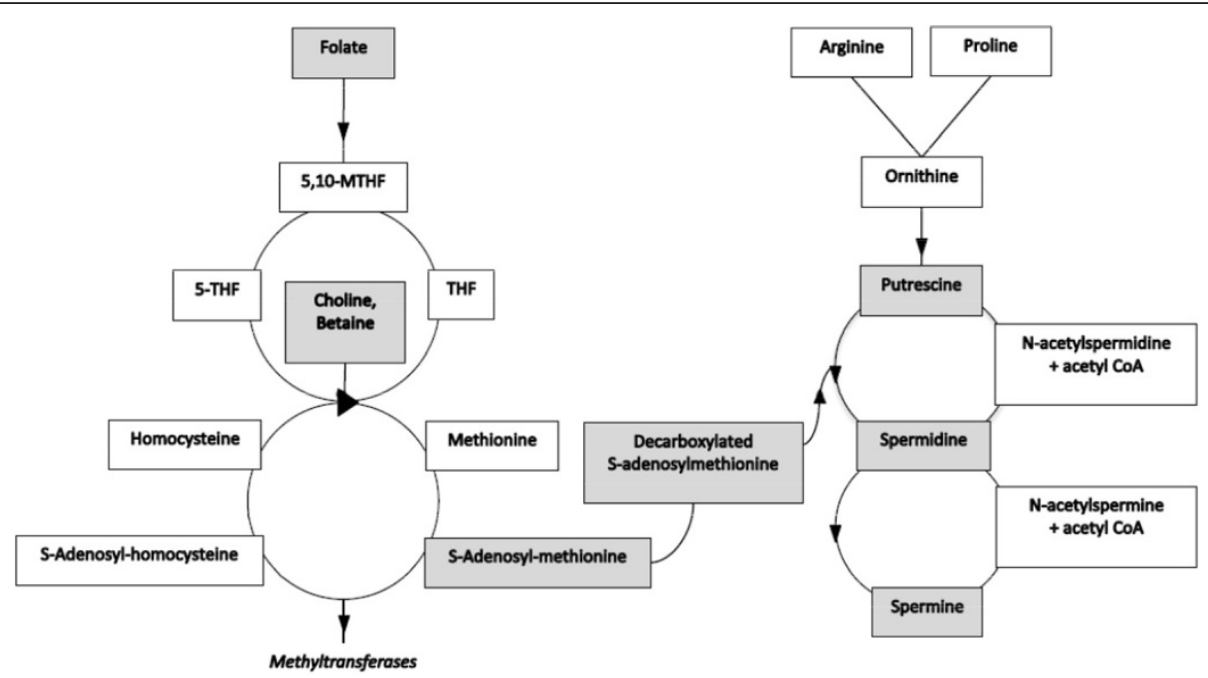

Figure 3 Folate, homocysteine and polyamine pathways. Gray cells highlight molecules of special interest. 
donor for methylation of nucleic acids, proteins, lipids and other molecules [9-11]. Methylation changes are the molecular basis for many imprinting [36] and some parentof-origin effects [37]. FA deficiency affects expression of many genes in mouse sperm [16]. Some FA-induced epigenetic changes can also be transmitted through the germline to affect phenotypic variation in subsequent generations [38-41]. Recently, the egg receptor (Juno) for the sperm cell-surface protein (Izumo1) was identified [42], see also [43]. These two proteins mediate egg-sperm recognition and activate a block to polyspermy. Interestingly, Juno is a member of a folate receptor family, but does not bind folate. Whether FA affects interactions between Juno and Izumo1 has not been tested. Anomalies in FA metabolism could therefore bias allelic inheritance through imprinting and related parent-of-origin effects, but direct evidence for effects on gametes and fertilization is lacking.

An alternative hypothesis involves polyamine metabolism. This pathway plays a central role in cell proliferation, cellular reprogramming, autophagy, transcription and translation, apoptosis and necrosis not only in somatic cells but also in haploid gametes [44,45]. Polyamines such as spermine, spermidine, putrescine and cadaverine are short chain organic molecules that possess several amines. Polyamines are highly charged molecules, with more than $90 \%$ of intracellular molecules bound to DNA and RNA. Their biosynthesis is one of the most highly regulated pathways in part because excess or deficiency can disrupt essential biological functions and because several end-products can be toxic [44,45]. SAM is both the methyl donor for all methylation reactions as well as the substrate for spermine and spermidine biosynthesis (Figure 3). When FA and SAM are limiting, cells preserve polyamine synthesis at the expense of methylation $[46,47]$. Acetyl-CoA is a co-factor in polyamine degradation. Acetyl-CoA is also used to produce choline and betaine, which serve as an alternative methyl donor (Figure 3). Thus anomalies in polyamine metabolism could affect methylation by limiting access to alternative methyl donors and by preferentially using SAM for polyamine biosynthesis rather than for methylation.

Polyamines play a prominent role in fertility and gamete function. Anomalies in polyamine levels are associated with infertility $[47,48]$ and dietary supplementation with SAM at least partially restores fertility [49]. Mice with transgenic over-expression of ornithine decarboxylase (ODC) are infertile [50,51]. ODC catalyzes the first reaction in synthesis of putrescine from arginine and proline; putrescine in turn is converted to spermidine and then spermine. Polyamine activity in spermatids and spermatozoa is tightly regulated [44]. OAZ3 (ornithine decarboxylase antizyme 3 - an ODC inhibitor) is a testis-specific inhibitor of ODC1 - the rate limiting step in polyamine synthesis. OAZ3 deficient mice produce aberrant sperm that are incapable of fertilization because of defects in sperm motility [52]; OAZ3 is a potent inhibitor of ODC in spermiogenesis [53,54]. Moreover, AZIN2, which blocks the inhibitory effects of OAZ3 on ODC, is abundant in haploid cells $[55,56]$. Gene expression profiles of Lrp6-deficient versus wild-type mice on control versus FA-supplemented diets show differences for several genes involved in polyamine synthesis, namely $\mathrm{Odcl}$, Sat1 - spermine/spermidine N-acetyl N1-transferase 1, and Oaz1 - ornithine decarboxylase antizyme 1 - another ODC inhibitor [24]. A recent study identified Oaz1 as a differentially expressed mRNA in sperm from folate deficient mice [16]. Thus folate supplementation in certain NTD mutant mice could compromise gamete function through either methylation metabolism, polyamine biology, or both.

In summary, FA exposure led to a strong departure from Mendelian segregation, with greatly reduced numbers of mutant heterozygotes and homozygotes without changes in embryonic viability. We propose that FA supplementation in these NTD models disrupted the folate, methylation and polyamine pathways, leading to preferential fertilization and biased segregation. Surveys are needed to test for similar results among dietary responses to FA supplementation with other NTD models to determine whether similar functions and pathways are involved. Effects of FA and polyamine supplementation on gamete function and fertilization should be tested in vivo and in vitro. Finally, studies are needed to test hypotheses about the developmental and biochemical mechanisms by which dietary supplements affect NTDs, embryonic viability, gamete biology, and fertilization.

\section{Conclusions}

In both humans and mouse models, dietary folate supplementation reduces the incidence and severity of neural tube defects, presumably by correcting developmental defects in the neural tube during embryogenesis. Tests for folate responsiveness in two NTD mouse models (in $A p o b^{\text {tm1Unc }}$ and $\operatorname{Vangl2^{Lp}}$ ) did not show a change in incidence or severity of NTDs, suggesting that these mutant mice are examples of NTD resistance to folate supplementation. Unexpectedly however we noted a biased Mendelian genotype distribution that strongly favored wild-type heterozygotes and homozygotes over mutant homozygotes. A review of the literature revealed other examples with similar biases, but these were interpreted as evidence for folate-induced embryonic lethality. Reanalysis of our results and published evidence revealed no evidence for reduced litter sizes or increased fetal resorptions in these cases. We propose that folic acid supplementation biases fertilization in favor of wild-type gametes, perhaps 
through folate-induced disturbances in polyamine metabolism.

\section{Methods}

Mice

Apob (B6.129P2-Apobtm1Unc/J; JR002053) and Lp (LPT/Le; JR000220) mutants were purchased from the Jackson Laboratory. All mice were raised on the PMI Nutrition Laboratory Autoclavable Rodent Diet \#5010 and maintained with trio matings. Test and control studies were contemporaneous.

\section{Study design}

Heterozygous males and females from both mutants were weaned at 3 weeks of age and thereafter maintained on either a control diet containing 2 ppm FA (D05072702, Research Diets) or a supplemented diet containing $10 \mathrm{ppm}$ FA (D05072701, Research Diets) for at least 3 weeks prior to mating (Figure 4). Timed pregnancies were then generated by mating $6-10$ week old females with males overnight. Upon discovery of a plug, females were kept on the same diet until they were sacrificed. Between E12.5-14.5 pregnant females were sacrificed and embryos examined (Figure 4). Tissues were obtained from all embryos for DNA extraction and genotyping. All mice shared the same animal room with controlled temperature, humidity, and 12 hour light-dark cycle. Mice were provided food and water ad libitum. The CWRU Institutional Animal Care and Use Committee approved all procedures.

\section{Special diets}

The only difference between the two diets used for the supplementation study was the amount of FA, which was 5 times higher in the supplemented diet $(10 \mathrm{ppm}$; D05072701, Research Diets) than the control diet (2 ppm; D05072702, Research Diets). We used 2 ppm FA because FA is required for proper breeding and fetal development based on many factors [57]. The estimated minimal FA requirement in mice is $0.5 \mathrm{ppm}$. However this concentration does not include a margin of safety [57]. Any concentration added to the diet should be higher than this minimum to account for nutrient losses during preparation and storage of the diet. In addition, a study similar to ours showed that $0 \mathrm{ppm}$ FA caused embryonic lethality of crooked-tail mutant embryos, but a shift to the expected exencephalic phenotype at $4 \mathrm{ppm}$ [33]. The percentage of affected $\mathrm{Cd} / \mathrm{Cd}$ embryos decreased with higher concentrations of FA (7 ppm or $10 \mathrm{ppm}$ ), indicating that $4 \mathrm{ppm}$ could serve as the control diet, thereby enabling the expected penetrance of NTDs, which was not possible with $0 \mathrm{ppm}$. At least two other studies have used this $2 \mathrm{ppm}$ versus $10 \mathrm{ppm}$ diet protocol $[24,25]$.

\section{Phenotype assessment}

Between E12.5-14.5, pregnant females were sacrificed and the embryos examined for NTDs, looped tail and resorptions. Resorptions were counted as dead embryos, including those that appeared only as 'dark spots' (necrosis) in the uterus. Resorptions were not genotyped. Litter size was counted as the number of live embryos at autopsy. The number of corpora lutea was not counted.

\section{Genotyping}

Genotyping for Apob was done according to the protocol provided by the Jackson Laboratory. The $L p$ genotyping protocol was previously described [58].

\section{Statistical analysis}

Statistical comparisons using the chi-square and Fisher's exact tests, as appropriate, were performed using GraphPad QuickCalcs Web site: http://graphpad.com/quickcalcs/ chisquared1.cfm and http://graphpad.com/quickcalcs/ contingency1.cfm.

Fisher's exact test was used to evaluate differences in the incidence of NTDs in mutant homozygotes between the $2 \mathrm{ppm}$ versus $10 \mathrm{ppm}$ diets (Table 1). Chi-square goodness-of-fit tests $(2 \mathrm{df})$ were used to test for departures from Mendelian expectations for the two NTD models on the $2 \mathrm{ppm}$ or $10 \mathrm{ppm}$ diets (Tables 2 and 3). We used both the statistical P value as well as the magnitude of the phenotype effect to assess results.

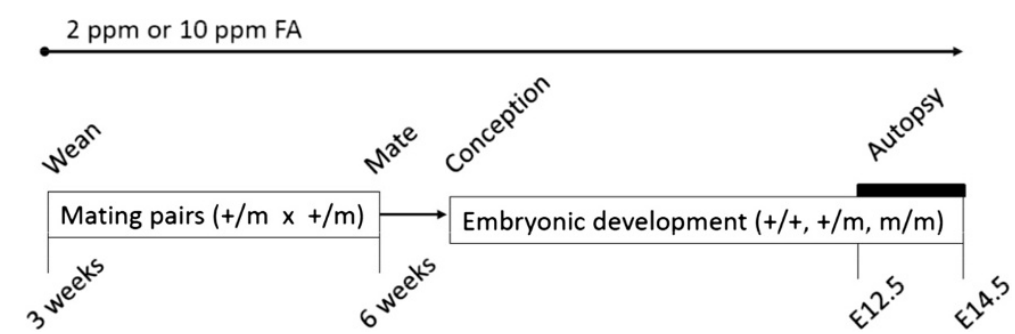

Figure 4 Dietary supplementation protocol. Three-week old female and male heterozygous mice ( or $L p$ mutants) were weaned on either the 2 ppm or 10 ppm FA diet, mated at 6 weeks of age, and then maintained on these diets through the remainder of the study. Embryos examined at E12.5 - E14.5. 


\section{Competing interests}

The authors declare no competing interests.

\section{Authors' contributions}

GAN performed the work, GAN and JHN conceived the study, planned the work, analyzed the data, and wrote the paper. Both authors read and approved the final manuscript.

\section{Acknowledgements}

NIH grants NS058979 and DP1HD075624 supported this work. We thank Mary Ann Handel, Marisa Bartolomei, Jacquetta Trasler, Betsy Ross, Lee Niswander, the Nadeau lab for thoughtful discussions of these problems, and the reviewers for their helpful comments and suggestions.

\section{Author details}

${ }^{1}$ Department of Genetics, Case Western Reserve University School of Medicine, Cleveland, OH, USA. ${ }^{2}$ Present address: Center for Human Genetics, University Hospitals, Cleveland, OH, USA. ${ }^{3}$ Present address: Pacific Northwest Research Institute, 720 Broadway, Seattle, WA 98122, USA.

Received: 11 June 2014 Accepted: 11 August 2014

Published: 27 August 2014

\section{References}

1. Detrait ER, George TM, Etchevers HC, Gilbert JR, Vekemans M, Speer MC: Human neural tube defects: developmental biology, epidemiology, and genetics. Neurotoxicol Teratol 2005, 27:515-524.

2. Copp AJ, Greene NDE: Neural tube defects - disorders of neurulation and related embryonic processes. WIRES Dev Biol 2013, 2:213-227.

3. Botto LD, Moore CA, Khoury MJ, Erickson JD: Neural-tube defects. N Engl J Med 1999, 341:1509-1519.

4. Northrup H, Volcik KA: Spina bifida and other neural tube defects. Curr Probl Pediatr 2000, 30:313-332.

5. Blom HJ, Shaw GM, den Heijer M, Finnell RH: Neural tube defects and folate: case far from closed. Nat Rev Neurosci 2006, 7:724-731.

6. Copp AJ, Stanier P, Greene NDE: Neural tube defects: recent advances, unsolved questions, and controversies. Lancet Neurol 2013, 12:799-810

7. Prevention of neural tube defects: results of the Medical Research Council Vitamin Study. MRC Vitamin Study Research Group. Lancet 1991 338:131-137.

8. Antony AC, Hansen DK: Hypothesis: folate-responsive neural tube defects and neurocrestopathies. Teratology 2000, 62:42-50.

9. Stover PJ: Polymorphisms in 1-carbon metabolism, epigenetics and folate-related pathologies. J Nutrigenet Nutrigenomics 2011, 4:293-305.

10. Salbaum JM, Kappen C: Genetic and epigenomic footprints of folate. Prog Mol Biol Transl Sci 2012, 108:129-158.

11. Guéant JL, Namour F, Guéant-Rodriguez RM, Daval JL: Folate and fetal programming: a play in epigenomics? Trends Endocrinol Metab 2013, 24:279-289.

12. Forges T, Pellanda H, Diligent C, Monnier P, Gueant J-L: Do folates have an impact on fertility? Gynecol Obstretr Fertil 2008, 36:93-939.

13. Laanpere M, Altmae S, Stavreus-Evers A, Nilsson TK, Yngve A, Salumets A Folate-mediated one-carbon metabolism and its effect on female fertility and pregnancy outcome. Nutr Rev 2009, 68:99-113.

14. Burdge GC, Lillycrop KA: Folic acid supplementation in pregnancy: are there devils in the detail? Br J Nutr 2012, 108:1924-1930.

15. Singh K, Jaiswal D: One-carbon metabolism, spermatogenesis, and male fertility. Reprod Sci 2013, 20:622-630.

16. Lambrot R, Xu C, Saint-Phar S, Chountalos G, Cohen T, Paquet M, Suderman M, Hallett M, Kimmins S: Low paternal dietary folate alters the mouse sperm epigenome and is associated with negative pregnancy outcomes. Nat Commun 2013, 4:2889.

17. Steegers-Theunissen RP, Twigt J, Pestinger V, Sinclair KD: The periconceptional period, reproduction and long-term health of offspring: the importance of one-carbon metabolism. Hum Reprod Update 2013, 19:640-655.

18. Li D, Pickell L, Liu Y, Wu Q, Cohn JS, Rozen R: Maternal methylenetetrhydrofolate reductase deficiency and low dietary folate lead to adverse reproductive outcomes and congenital heart defects in mice. Am J Clin Nutr 2005, 82:188-195.
19. Pickell L, Brown K, Li D, Wang X-L, Deng L, Wu Q, Selhub J, Luo L, Jerome-Majewska L, Rozen R: High intake of folic acid disrupts embryonic development in mice. Birth Defects Res (Part A) 2011, 91:8-19.

20. Mikael LG, Deng L, Paul L, Selhub J, Rozen R: Moderately high intake of folic acid has a negative impact on mouse embryonic development. Birth Defects Res (Part A) 2013, 97:47-52.

21. Greene NDE, Copp AJ: Mouse models of neural tube defects: investigating preventative mechanisms. Am J Med Genet 2005, 135C:1-41.

22. Zohn IE: Mouse as a model for multifactorial inheritance of neural tube defects. Birth Defects Res Part C 2012, 96:193-205.

23. Woldarczyk BJ, Tang LS, Triplett A, Aleman F, Finell RH: Spontaneous neural tube defects in splotch mice supplemented with selected micronutrients. Toxicol Appl Pharmacol 2006, 213:55-63.

24. Gray JD, Nakouzi G, Slowinska-Castaldo B, Dazard J-E, Rao JS, Nadeau JH, Ross ME: Functional interactions between the LRP6 WNT co-receptor and folate supplementation. Hum Mol Genet 2010, 19:4560-4572.

25. Marean A, Graf A, Zhang Y, Niswander L: Folic acid supplementation can adversely affect murine neural tube closure and embryonic survival. Hum Mol Genet 2011, 20:3678-3683.

26. Harris MJ, Juriloff DM: An update to the list of mouse mutants with neural tube closure defects and advances toward a complete genetic perspective of neural tube closure. Birth Defects Res (Part A) 2010, 88:653-669.

27. Homanics GE, Smith TJ, Zhang SH, Lee D, Young SG, Maeda N: Targeted modification of the apolipoprotein $B$ gene results in hypobetalipoproteinemia and developmental abnormalities in mice. Proc Natl Acad Sci U S A 1993, 90:2389-2393.

28. Juriloff DM, Harris MJ: Animal models of neural tube defects. Mental Retard Dev Disabil Res Rev 1998, 4:254-263.

29. Torban E, Wang HJ, Groulx N, Gros P: Independent mutations in mouse Vangl2 that cause neural tube defects in looptail mice impair interaction with members of the Dishevelled family. J Biol Chem 2004, 279:52703-52713.

30. Greene ND, Gerrelli D, Van Straaten HW, Copp AJ: Abnormalities of floor plate, notochord and somite differentiation in the loop-tail (Lp) mouse: a model of severe neural tube defects. Mech Dev 1998, 73:59-72.

31. Carter M, Ulrich S, Oofuji Y, Williams DA, Ross ME: Crooked tail (Cd) models human folate-responsive neural tube defects. Hum Mol Genet 1999, 8:2199-2204.

32. Ernest $\mathrm{S}$, Carter M, Shao H, Hosack A, Lerner N, Colmenares C, Rosenblatt DS, Pao YH, Ross ME, Nadeau JH: Parallel changes in metabolite and expression profiles in crooked-tail mutant and folate-reduced wild-type mice. Hum Mol Genet 2006, 15:3387-3393.

33. Leung K-Y, de Castro SCP, Savery D, Copp AJ, Greene NDE: Nucleotide precursors prevent folic acid-resistant neural tube defects in the mouse. Brain 2013, 136:2836-2841.

34. Momb J, Lewandowski JP, Bryant JD, Fitch R, Surman DR, Vokes SA, Appling DR: Deletion of Mthfd1I causes embryonic lethality and neural tube and craniofacial defects in mice. Proc Natl Acad Sci U S A 2013, 110:549-554.

35. Chan D, Cushnie DW, Neaga OR, Larance AK, Rozen R, Trasler JM: Strain-specific defects in testicular development and sperm epigenetic patterns in 5,10-methylenetetrahydrofolate-deficient mice. Endocrinology 2010, 151:3363-3373.

36. Lee JT, Bartolomei MS: X-inactivation, imprinting, and long noncoding RNAs in health and disease. Cell 2013, 152:1308-1323.

37. Mott R, Yuan W, Kaisaki P, Gan X, Cleak J, Edwards A, Baud A, Flint J: The architecture of parent-of-origin effects in mice. Cell 2014, 156:332-342

38. Wolff GL, Kodell RL, Moore SR, Cooney CA: Maternal epigenetics and methyl supplements affect agouti gene expression in Avy/+mice. FASEB J 1998, 12:949-957.

39. Blewitt ME, Vickaryous NK, Paldi A, Koseki H, Whitelaw E: Dynamic reprogramming of DNA methylation at an epigenetically sensitive allele in mice. PLoS Genet 2006, 2:e49.

40. Cropley JE, Suter CM, Beckman KB, Martin DI: Germ-line epigenetic modification of the murine Avy allele by nutritional supplementation. Proc Natl Acad Sci U S A 2006, 103:17308-17312.

41. Padmanabhan N, Jia D, Geary-Joo C, Wu X, Ferguson-Smith AC, Fung E, Bieda MC, Snyder FF, Gravel RA, Cross JC, Watson ED: Mutation in folate metabolism causes epigenetic instability and transgenerational effects on development. Cell 2013, 155:81-93.

42. Bianchi E, Doe B, Goulding D, Wright GJ: Juno is the egg Izumo receptor and is essential for mammalian fertilization. Nature 2014, 508:483-487. 
43. McKnight K, Hoang HD, Prasain JK, Brown N, Vibbert J, Hollister KA, Moore $R$, Ragains JR, Reese J, Miller MA: Neurosensory perception of environmental cues modulates sperm motility critical for fertilization. Science 2014, 344:754-757.

44. Lefevre PLC, Palin M-F, Murphy BD: Polyamines on the reproductive landscape. Endocrine Rev 2011, 32:694-712.

45. Bauer MA, Carmona-Gutierrez D, Ruckenstuhl C, Reisnbichler A, Magalou EV, Eisenberg T, Magnes C, Jungwirth H, Sinner FM, Pieber TR, Frohlich K-U, Kroemer G, Tavernarakis M, Madeo F: Spermidine promotes mating and fertilization efficiency in model organisms. Cell Cycle 2013, 12:346-352.

46. Bistulfi G, Diegelman P, Foster BA, Kramer DL, Porter CW, Smiraglia DJ: Polyamine biosynthesis impacts cellular folate requirements necessary to maintain S-adenosylmethionine and nucleotide pools. FASEB J 2009, 23:2888-2897.

47. Sun D, Wollin A, Stephan AM: Moderate folate deficiency influences polyamine synthesis in rats. J Nutr 2002, 132:2632-2637.

48. Shoat B, Maayan R, Singer R, Sagiv M, Kaufman H, Zukerman Z: Immunosuppressive activity and polyamine levels of seminal plasma in azospermic, oligospermic, and normospermic men. Arch Androl 1990, 24:41-50.

49. Calandra RS, Rulli SB, Frungieri MB, Suescun MO, Gonzalez-Calvar SI: Polyamines in the male reproductive system. Acto Physiol Pharmacol Ther Latinoam 1996, 46:209-222.

50. Vanella A, Pinturo R, Vasta M, Piazza G, Rapisarda A, Savoca S, Nardo F, Bellia $\checkmark$, Panella M: Polyamine levels in human semen of unfertile patients: effect of S-adenosylmethionine. Acta Eur Fertil 1978, 9:99-103.

51. Halmekytö M, Hyttinen JM, Sinervirta R, Utriainen M, Myöhänen S, Voipio HM, Wahlfors J, Syrjänen S, Syrjänen K, Alhonen L: Transgenic mice aberrantly expressing human ornithine decarboxylase gene. J Biol Chem 1991, 266:19746-19751.

52. Kilpelainen PT, Saarimies J, Kontusaari SI, Jarvinen MJ, Peralta Soler A Kallioinen MJ, Hietala OA: Abnormal ornithine decarboxylase activity in transgenic mice increases tumor formation and infertility. Int J Biochem Cell Biol 2001, 33:507-520.

53. Tokuhiro K, Isotani A, Yokota S, Yano Y, Oshio S, Hirose M, Wada M, Fujita K, Ogawa Y, Okabe M, Nishimune Y, Tanaka H: OAZ-t/OAZ3 is essential for rigid connection of sperm tails to heads in mouse. PLoS Genet 2009, 5:e10000712.

54. Qian ZU, Tsai YH, Steinberger A, Lu M, Greenfield A, Haddox MK: Localization of ornithine decarboxylase in rat testicular cells and epididymal spermatozoa. Biol Reprod 1985, 33:1189-1195.

55. Pitkanen LT, Heiskala, Anderson LC: Expression of a novel ornithine decarboxylase-like protein in the central nervous system and testes. Biochem Biophys Res Commun 2001, 287:1051-1057.

56. Lopez-Contreras AJ, Ramos-Molina B, Martinez-de-la-Torre M, Penafil-Verdu C, Puelles L, Cremades A, Peñafiel R: Expression of antizyme inhibitor 2 in male haploid germinal cells suggests a role in spermiogenesis. Int J Biochem Cell Biol 2009, 41:1070-1078.

57. National Academies Press: Nutrient requirements of the mouse. In Nutrient Requirements of Laboratory Animals. Fourth Revisedth edition. Washington, DC: National Academy Press; 1995:80-102.

58. Copp AJ, Checiu I, Henson JN: Developmental basis of severe neural tube defects in the loop-tail (Lp) mutant mouse: use of microsatellite DNA markers to identify embryonic genotype. Dev Biol 1994, 165:20-29.

doi:10.1186/s12863-014-0091-x

Cite this article as: Nakouzi and Nadeau: Does dietary folic acid

supplementation in mouse NTD models affect neural tube development or gamete preference at fertilization? BMC Genetics 2014 15:91.

\section{Submit your next manuscript to BioMed Central and take full advantage of:}

- Convenient online submission

- Thorough peer review

- No space constraints or color figure charges

- Immediate publication on acceptance

- Inclusion in PubMed, CAS, Scopus and Google Scholar

- Research which is freely available for redistribution

Submit your manuscript at www.biomedcentral.com/submit
C Biomed Central 